\title{
La charte de l'éthique des facultés de médecine : un cadre conceptuel et un outil
}

De longue date, la démarche éthique des facultés de médecine était l'objet de discussions souvent informelles mais passionnées lors des réunions de la Conférence Internationale des $D$ oyens et des Facultés de M édecine d'Expression Française (CIDMEF) et plus particulièrement au sein de son conseil d'administration (le bureau permanent). II sagissait, tout autant, d'une préoccupation de responsables universitaires venant des pays « nantis » que des pays en dével oppement. Le Professeur M oïse Oliveira, ancien Recteur de I'U niversité de Libreville, et le Professeur Abdelaziz Ghachem, ancien Doyen de la Faculté de $M$ édecine deTunis, ne manquaient jamais de rappeler l'importance qu'il y aurait à affirmer dansle monde universitaire francophone médical la valeur de l'éthique. Cette quête axiologique est une constante de la CIDM EF depuis sa fondation en 1981. Les principes humanistes qui guident cette organisation internationale sont exposés dans le récent livre de son directeur général, le Professeur André Gouazé, avec une préface remarquable de Monsieur Abdou Diouf, secrétaire général de I'O rganisation Internationale de la Francophonie et ancien Président de la République du Sénégal ${ }^{1}$. U ne préoccupation de la CID M EF était la mise en cuvre d'une réflexion sur l'éthique comparée entre les grandes zones géographiques et les pays qui les composent ( $M$. Sosso, Vice Recteur de l'U niversité de Yaoundé - Cameroun et $A$. Gouazé- Tours).

D'autres influences s'avéraient nécessaires pour que la Charte de l'éthique des facultés de médecine soit mise en chantier. II sagi ssait tout d'abord de la démarche d'assurance qualité de I'enseignement médical promue par I'O rganisation M ondiale de la Santé (OMS). L'objectif de la qualité d'éducation médicale ( «D octeur 5 étoil les») a été une préoccupation de l'OM S2. Cet organisme a mis en avant plus récemment la nécessité d'une responsabilité sociale des facultés de médecine. A ce titre, on peut parler d'une véritable " professionnalisation desfacultés de médecine» avec lemêmesens que celui utilisé pour les hommes (professionnalisme des enseignants de méded ne, professionnalisme des futurs médecins). La professionnalisation dela facultédemédecineavait déà été prônéeil y a plusieurs années dans un article sur les enjeux et les risques de la formation initiale des médecins dans les années futures ${ }^{4}$. D ès lors, il ne sagissait plus d'une smple démarchequalité mais d'une véritable assurance qualité del'éducation médicale. La faculté de médecine se doit non seulement d'assurer la qualité des médecins qu'elle forme mais aussi de collaborer efficace ment avec la société L'éthique d'une faculté de médecine se dédine à différents niveaux : d'une part, assurer la qualité professionnelle des étudiants qu'elle forme ; d'autre part, sassurer qu'elle partici pe avec les autres partenaires de la santé (décideurs politiques, professions de santé, communauté et gestionnaires de la santé) à une production de services répondant aux gens ${ }^{5}$. II sagit de l'approche « vers |'unité pour la santé » (VUPS) ${ }^{6}$.

II y avait nécessité de mettre en place un cadre de réflexion sur l'éthique des pratiques d'enseignement dans les facultés de médecine comme l'a bien montré A. Quinton ${ }^{7}$ parlant de manquements à I'éthiqueà de multiples niveaux. II parle des grandes injures qui peuvent être faites à l'éthique dans l'accuél et la sélection des étudiants (autochtones ou étrangers), avec des problèmes potentiels de racisme, de sexisme, de harcèlement en milieu universitaire. II donne des références sur le recours aux psychostimulants pour les examens et aux concours. L'absence de contrat clair entre la faculté, l'enseignant et l'étudiant est bien souvent un manquement à l'éthique. Les manquements à l'éthique dans la 
recherche et dans les contenus de l'enseignement (rigueur scientifique) sont évoqués ; citons enfin les conflits d'intérêt entre les acteurs de l'enseignement et les manquements à l'éthique concernant la finalité de la faculté (ses missions et ses fonctions).

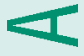

La perte de sensibilité morale des étudiants en médecine savère être une autre préoccupation pouvant faire parler d'une véritable urgence ${ }^{\beta, 9}$ pour l'implantation d'un programme de formation au professionnalisme des futurs médecins ${ }^{10}$.

Tout ceci amenait la CIDMEF à décider à Tours en 2001 la rédaction d'une charte de l'éthique des facultés de médecine.
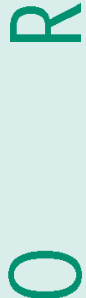

Son objectif n'était pas de légiférer, de mettre en place des règles contraignantes en dehors desquelles une faculté de médecine n'était plus crédible. II sagissait au contraire de bâtir un cadre conceptuel permettant à chaque établis sement del'enseignement supérieur médical de réfléchir et d'améliorer ses pratiques ; d'ailleurs, la charte de l'éthique des facultés de médecine n'a rien d'un outil réglementaire définitif. Elle est susceptible d'être améliorée et enrichie tant au sein de la Conférence Internationale que des Conférences dites Régionales (Q uébec, France, M aghreb, AfriqueSubsaharienne, etc. ). Chaque établis sement peut de lui-même la faire évoluer. Le texte est un dénominateur commun (leplus petit, mais ce terme est-il approprié ?). Certains domaines de la charte ont été l'objet de discussions vives : citons celui de la marchandisation des études supérieures ${ }^{11}$ ou celui de la participation (de la collaboration ?) des malades à l'enseignement et à la recherche.

La réflexion sorganise déà dans le monde francophone et en dehors. II est souhaité des traductions dans le maximum deslanguesutilisées dans nos facultés (des traductions en anglais, italien, vietnamien, allemand, etc sont en cours).

La traduction en arabe classique est déjà sur le site de la CIDMEF (http://www.cidmef. u-bordeaux2.fr/).

La francophonie setourneversle reste du monde non pas pour lui imposer un standard mais pour lui proposer un partage de valeurs Aucun droit de publication ne sera exigé dans quelque langue que ce soit. Aucune autorisation de publier n'est exigible. Améliorer la diffusion de ce texte nous a éé facilitée par le D octeur Jean $\mathrm{N}$ au (AVEN TIS) qui a facilité la publication d'une monographie "La Faculté de médecine face à ses responsabilités sociales et éthiques», rédigée en collaboration avec I'OMS, où la Charte de l'éthique des facultés de médecine figure en bonne place ${ }^{12}$. II est prévu un texte court reprenant les diverses thématiques et pouvant être affiché ici dansune faculté de médedne, là dans un service hospitalier. La stratégie devant amener une réflexion constructive dans les facultés de médecine n'est pas simple car le mot éthique peut faire peur ou être suspect d'intellectualisme utopique. C'est à l'aune des expériences concrètes institutionnelles que l'on pourra mesurer l'influence de ce texte. Cela se fera " chemin faisant » car partir d'un cadre théorique pour aller à des considérations pratiques et à des actes est un cheminement difficile où chaque facultén'ira pas du même pas.

Un autre débat samorce au niveau international : celui de l'évaluation des programmes des facultés de médecine. L'opération menée par le conseil d'évaluation de la CIDMEF a permis d'évaluer de nombreux établissements du $\mathrm{N}$ ord comme du Sud. Les recommandations tirées des constats de manquements à l'éthique vont pouvoir maintenant sappuyer sur un cadre référentiel. M ais... qu'en sera-t-il lorsque se mettra en place une accrédition des facultés de médecine?

JacquesH enri BARRIER mailto:J acques.Barrier@santeuniv-nantesfr Lucie BRAZEAU-LAM ON TAGNE mailto:Lucie.Brazeau@U Sherbrooke.ca 


\section{Références}

1. Gouazé A. Abdelaziz, Moumouni, Petru, Trung, Pierre et les autres... ou une certaine vision de la médecine et de la santé dans le monde francophone. Paris : Ed. Expansion Scientifique, 2004.

2. Boelen C. M edical education reforms : the need for global action. Acad $M$ ed $1992 ; 67: 11-16$.

3. Boelen $C, H$ eck JE. D éfinir et mesurer la responsabilité sociale des Facultés de M édecine. G enève : Ed O M S, 2000.

4. Barrier JH. Enjeux et risques de la formation initiale des médecins dans les années futures. Ann M ed Interne 2001 ; $152: 491-493$.

5. Boelen C. Facultés de M édecine et santé de la communauté. In : La faculté de médecine face à ses responsabilités sociales et éthiques. JH Barrier et J Roland ( $\mathrm{Eds})$. U niversités de $\mathrm{N}$ antes et de N ancy, 2004.

6. Towards unity for health : opportunities and challenges for partnership in health development. G eneve : Ed OM S, 2000.

7. Quinton A. Proposition d'un cadre de réflexion sur l'éthique des pratiques d'enseignement dans les Facultés de $M$ édecine. In : La faculté de médecine face à ses responsabilités sociales et éthiques. JH Barrier et J Roland (Eds). Universités de Nantes et de Nancy, 2004.
8. Hebert PC, Meslin EM, Dunn EV. $M$ easuring the ethical sensitivity of medical students : a study at the University of Toronto. J M ed Ethics 1992 ; 18 : 142-147.

9. Barrier $J H$, Brazeau-Lamontagne $L$, Pottier $P$, Boutoille $D$. Compétences éthiques des étudiants en médecine durant leur stage en médecine interne. Rev M ed Interne, sous presse.

10. Barrier $J H$, Brazeau-Lamontagne $L$, Colin R, Q uinton A, Llorca G, Ehua FS. La formation au professionnalisme des futurs médecins.R ecommandations du Conseil Pédagogique de la CID MEF. Pédagogie M édicale 2004 ; 5 : 75-81.

11. L'U niversité américaine vampirisée par les marchands; en France, la douce trahision des clercs. Le Monde D iplomatique, mars 2001 : 20-21.

12. La faculté de médecine face à ses responsabilités sociales et éthiques. JH Barrier et J Roland (Eds). Universités de $\mathrm{N}$ antes et de N ancy, 2004. 